\title{
A novel aspect of the structure of the avian thymic medulla
}

\author{
Ildikó Bódi • Krisztina Minkó • Dávid Molnár • \\ Nándor Nagy • Imre Oláh
}

Received: 4 December 2013 / Accepted: 7 October 2014 /Published online: 9 November 2014

(C) Springer-Verlag Berlin Heidelberg 2014

\begin{abstract}
We provide evidence for the compartmentalization of the avian thymic medulla and identify the avian thymic dendritic cell. The thymic anlage develops from an epithelial cord of the branchial endoderm. Branches of the cord are separated by primary septae of neural crest origin. The dilation of the primary septae produces the keratin-negative area (KNA) of the thymic medulla and fills the gaps of the keratin-positive network (KPN). Morphometric analysis indicates that the KNA takes up about half of the volume of the thymic medulla, which has reticular connective tissue, like peripheral lymphoid organs. The KNA receives blood vessels and in addition to pericytes, the myoid cells of striated muscle structure occupy this area. The myoid cells are of branchial arch or prechordal plate origin providing indirect evidence for the neural crest origin of the KNA. The marginal epithelial cells of the KPN co-express keratin and vimentin intermediate filaments, which indicate their functional peculiarity. The basal lamina of the primary septum is discontinuous on the surface of the KPN providing histological evidence for the loss of the blood-thymus barrier in the medulla. In the center of the KNA, the dendritic cells lie in close association with blood vessels, whereas the B-cells accumulate along the KPN. The organization of the KPN and KNA increases the "surface" of the so-called cortico-medullary border, thereby contributing to the efficacy of central tolerance.
\end{abstract}

This work was supported by OTKA grant number 69061 and sponsored by Prophyl Kft., Mohács, Hungary.

I. Bódi $\cdot$ K. Minkó $\cdot$ D. Molnár · N. Nagy $\cdot$ I. Oláh $(\triangle)$

Department of Human Morphology and Developmental Biology,

Faculty of Medicine, Semmelweis University, Tüzoltó Str. 58,

Budapest 1094, Hungary

e-mail: olah.imre@med.semmelweis-univ.hu
Keywords Thymic medulla $\cdot$ Keratin-negative area . Keratin-positive network · Cortico-medullary border $\cdot$ Thymic dendritic cell $\cdot$ Chicken

\section{Introduction}

The hematoxylin-eosin (HE)-stained thymus is divided into a cortex of densely packed lymphocytes and a medulla with far fewer lymphocytes but with more epithelial cells having a bulky cytoplasm. The three-dimensional thymic epithelial reticulum develops from the third and fourth branchial pouch endoderm (Farley et al. 2013; Gill et al. 2003; Gordon and Manley 2011; Le Douarin 1967; Le Douarin et al. 1984; Neves et al. 2012; Rodewald 2008; Venzke 1952) and differentiates into cortical and medullary epithelial cells. Le Douarin (1967) isolated the branchial pouch endoderm, before fusion with the ectoderm of the branchial groove and co-cultured it with the mesenchyme; this resulted in the formation of functional thymic tissue providing evidence that (1) branchial endoderm without ectoderm was capable of thymic organogenesis and (2) thymic epithelial cell (TEC) differentiation required mesenchymal cell cooperation. The mesenchymal capsule surrounding the thymic rudiment, interlobular septae and the pericytes of the medulla develops from the cranial neural crest (Bockman and Kirby. 1984; Foster et al. 2008; Jenkinson et al. 2003; 2007; Le Lièvre and Le Douarin 1975; Müller et al. 2008; Noden 1978, 1983). Lack of a distinct mesenchymal cell population, which expresses platelet-derived growth factor receptor $\alpha(\operatorname{PDGFR} \alpha)$ results in diminished epithelial cell proliferation and subsequent hypoplastic thymus development (Jenkinson et al. 2007). Experiments of the last decade have confirmed not only the mesenchymal induction of TEC (Jenkinson et al. 2007; Itoi et al. 2007; Neves et al. 2012) but also that the epithelial-mesenchymal cross-talk is extended to epithelial-T-cell interaction (Klug et al. 2002; Rossi et al. 
2007). A common bipotent progenitor of TEC exists not only in the early embryo but also persists in the postnatal and possibly in adult thymus (Bennett et al. 2002; Bleul et al. 2006; Farr et al. 2002; Rossi et al. 2007).

Immature thymocytes colonize the subcapsular region of the thymus and during maturation, the single-positive $\mathrm{CD} 4^{+}$ and $\mathrm{CD}^{+}$cells enter the medulla. Thus, the cortical TEC is responsible for T-cell receptor development, the positive selection of double-positive cells and the differentiation of the single-positive thymocytes, whereas medullary TEC and dendritic cells (DCs) at the cortico-medullary border regulate and/ or execute negative selection by deleting the autoreactive $\mathrm{CD}^{+}$and $\mathrm{CD}^{+}$T-cells and subsequently generate central tolerance to self-antigen. The various functions of the cortical and medullary TECs possibly require different cytological phenotypes. At the 12th embryonic day, the putative progenitor marker, Mts24, is present in almost every epithelial cell (Rossi et al. 2007) and therefore, either the majority of the TECs can be considered to be bipotent progenitors or the Mts24 cannot be regarded as a specific progenitor marker. The latter statement seems to be true, because both Mts24positive and -negative cells are capable of producing functional thymic tissue that supports T-cell development (Rossi et al. 2007). Klug et al. (1998) detected two distinct TEC populations. The major one expresses keratin $(\mathrm{K}) 8$, whereas the minor subpopulation localizing at the cortico-medullary border produces both $\mathrm{K} 8$ and $\mathrm{K} 5$ intermediate filaments. The presence of the two intermediate filaments in TECs suggests two different functions and stages of differentiation. The relationship of the putative TEC progenitor marker, Mts24, to the keratin expression of cortical and cortico-medullary TEC in mammals remains unknown.

Previously, a keratin-negative area (KNA) has been known to occur in between the medullary TEC in mice (Van Ewijk et al. 1980) and in chicken (Boyd et al. 1992; Guillemot et al. 1984; Minkó and Oláh 1996). Although the cross-talk between the medullary TEC and mesenchyme has become generally accepted, the origin and role of the KNA of the thymic medulla remains unknown. The presence of KNA in the thymic medulla raises several questions that might be relevant for thymic function. (1) If the KNA is merely a dilation of the primary septum (PS) of neural crest origin, does it belong to the thymic medulla? (2) If so, is the medulla divided into a keratin-positive network (KPN) occupied by TEC and KNA, which develops from the neural crest cells, like the capsule and the septum themselves? (3) If the KNA is a part of the medulla, how can we determine the cortico-medullary border? (4) What is the function of the KPN and KNA? (5) Where are the DCs in the KPN and/or KNA? These questions are primarily related to anatomical or topographical problems but the lack of accurate morphological knowledge hinders the correct interpretation of the immunological function of the thymus.
The renaissance of thymic stromal cell research and our former observations (Minkó and Oláh 1996) have initiated the renewal of studying the thymic medulla. Our study indicates that, in the chicken, the volume of the KNA is close to $50 \%$ of the medulla, which consists of connective tissue and produces extracellular matrix (ECM), unlike the KPN and cortex. The KNA of the medulla is much larger than the perivascular space, which is continuous with the PS. Hemopoietic cells of the KNA show a highly organized pattern that might contribute to the efficacy of negative selection.

\section{Materials and methods}

Animals

White Leghorn (Gallus gallus) eggs and chickens were obtained from a commercial breeder. Eggs were incubated at $38{ }^{\circ} \mathrm{C}$ in a humidified incubator. Embryos were staged according to the number of days of incubation. Tissue samples were taken from five chicken embryos at each of the sampling days at 11,13,15, 16, 17 and 20 days and from five 6-weekold adult chickens. Thymuses from 8-week-old rabbit and rat were used to compare the keratin staining pattern in the medulla of the bird with that of mammals. The experimental design and the conditions of the animals were approved by the Animal Ethical Committee of Semmelweis University (Budapest, Hungary).

\section{Silver impregnation}

Silver impregnation was performed to demonstrate the reticular fibers. The thymic lobes from 6 -week-old chickens were fixed in $4 \%$ buffered paraformaldehyde, overnight. After being washed in phosphate-buffered saline (PBS, pH 7.4), tissue samples were embedded in paraffin and sectioned; the $8-\mu \mathrm{m}$-thick sections were deparaffinized. Silver impregnation was carried out according to Gomori (Krutsay 1980).

Immunohistochemistry and immunofluorescence

All the immunohistochemistry and immunofluorescence patterns presented here were obtained from at least five individual animals. For immunostaining, tissue samples were taken and completely covered by liver, from the same bird and the composite blocks were frozen in liquid nitrogen. Cryostat sections $(10 \mu \mathrm{m}$ thick) were mounted onto poly-L-lysine (Sigma Aldrich)-coated slides and fixed in cold acetone for $10 \mathrm{~min}$. The sections were rehydrated in PBS and the endogenous peroxidase activity was quenched with $3 \%$ hydrogen peroxide (Sigma-Aldrich) for $10 \mathrm{~min}$ or developed with 3,3diaminobenzidine (DAB, Sigma-Aldrich). Sections were washed in PBS and incubated with the primary antibodies 
(Table 1) for $45 \mathrm{~min}$ at room temperature. Incubation of primary antibodies was followed by biotinylated horse antimouse IgG (Vector Laboratories) or anti-rabbit IgG (Vector Laboratories) for $45 \mathrm{~min}$. The signal was enhanced by using the avidin-biotinylated peroxidase complex (ABC, Vector Laboratories) for $30 \mathrm{~min}$. Peroxidase activity was visualized with 4-chloro-1-naphtol (Sigma-Aldrich). The staining patterns of the various antibodies (anti-CD4, anti-CD8 and anticytokeratin) were compared on serial sections.

For double-immunofluorescent staining, sections were incubated with primary antibodies, followed by labeling with AlexaFluor594-conjugated goat anti-mouse IgG1 secondary antibodies (Invitrogen). The ZenonFluor488 IgG1 antibody labeling kit (Invitrogen) was used to tag the anti-keratin antibody. Cell nuclei were stained with 4,6-diamidino-2phenylindole (DAPI; Invitrogen). Sections were examined with fluorescence and confocal laser scanning microscopes (Zeiss Axiophot, Bio-Rad Radiance 2100 Rainbow, respectively). Digital images were captured with an AxioCam HRC camera and compiled by using Adobe Photoshop. Line drawings were made with Adobe Illustrator (Microsoft Office).

\section{Morphometric measurement}

The volumetric ratio of the thymic cortex and medulla and the ratio of the KPN and KNA of the medulla were studied by using the Cavalieri method and Neurolucida software (MBF Biosciences). The Cavalieri method is a stereological probe for quantifying regional volumes (Tschanz et al. 2014). The volume of the thymus was estimated by summing the areas and multiplying the result by the slice thickness. Cryostat sections (12 $\mu \mathrm{m}$ thick) from the thymic lobes of a 3-and a 6-week-old chicken were taken and every fiftieth and hundredth section, respectively, was immunostained with anti-cytokeratin. Seven and eight sections were morphometrically evaluated in 6- and 3-week-old chickens, respectively, representing the entire thickness of a thymic lobe.

\section{Electron microscopy}

Briefly, the tissue samples were fixed in $4 \%$ buffered glutaraldehyde solution overnight and post-fixed in $1 \%$ osmium tetroxide (Polysciences) for $2 \mathrm{~h}$. After dehydration in graded ethanol, the tissue blocks were embedded in Polybed/Araldite 6500 (Polysciences) mixture. For transmission electron microscopy, ultrathin sections were contrasted with uranyl acetate and lead citrate and studied with a Hitachi electron microscope, type H-7600.

\section{Dexamethasone treatment}

Ten 6-week-old chickens were treated with dexamethasone (DM; Ratiopharm Hungaria) to induce thymic involution and to check the alteration of the medullary KPN and KNA. DM at $5 \mathrm{mg} / 1000 \mathrm{~g}$ body weight was administered intraperitoneally for 10 consecutive days. Thymic lobes were removed $2 \mathrm{~h}$ after the last injection of DM.

\section{Results}

An epithelial cord grows out and divides from the third and fourth branchial pouch endoderm. Branches of the cord are

Table 1 Primary antibodies used

\begin{tabular}{|c|c|c|c|c|}
\hline Antibody/clone & Host & Antibody specificity & Dilution & Source of antibody \\
\hline Lu-5 & Mouse IgG1 & Pan-cytokeratin & $1: 100$ & BMA \\
\hline Basal cell cytokeratin & Mouse IgG1 & Cytokeratin 5 & $1: 200$ & Millipore \\
\hline AMF-17b & Mouse IgG1 & Vimentin & Supernatant & DSHB \\
\hline 3B4 & Mouse IgG2a & Vimentin & $1: 200$ & Millipore \\
\hline 31 & Mouse IgG1 & Laminin & $1: 50$ & DSHB \\
\hline $3 \mathrm{~B} 2$ & Mouse IgG1 & Collagen type III & $1: 1500$ & DSHB \\
\hline B3/D6 & Mouse IgG1 & Fibronectin & $1: 5$ & DSHB \\
\hline M1B4 & Mouse IgG1 & Tenascin & $1: 5$ & DSHB \\
\hline D76 & Mouse IgG1 & Desmin & $1: 4$ & DSHB \\
\hline CVI-ChNL-74.3 & Mouse IgG1 & Chicken dendritic cell marker & $1: 200$ & Prionics \\
\hline BoA1 & Mouse IgG1 & $\operatorname{chB6}$ & Supernatant & Igyártó et al. (2008) \\
\hline CT4 & Mouse IgG1 & Chicken CD4 & $1: 200$ & Southern Biotech. \\
\hline CT8 & Mouse IgG1 & Chicken CD8 & $1: 200$ & Southern Biotech. \\
\hline $1 \mathrm{~A} 4$ & Mouse IgG2a & $\alpha$-Smooth muscle actin & $1: 200$ & Dako \\
\hline TAP1 & Mouse IgG2a & Antigen-presenting cell & $1: 200$ & DSHB \\
\hline
\end{tabular}


separated from one another by PSs, which receive the thymic arteries and veins. The first histological sign of the formation of the thymic medulla appears on the 15th-16th days of incubation. Cytokeratin immunohistochemistry reveals slightly elevated keratin staining in close association with the PS, which serves as a stalk for the formation of KNA (Fig. 1a). These increased keratin staining spots indicate the anlage of the future KPN of the thymic medulla (Fig. 1a, b). Each branch of the epithelial cord ends in a club-shaped enlargement, which is the rudiment of the cortex (Fig. 1b). The secondary septae (SSs) develop later in the club-shaped enlargement, they are narrower than the PSs, do not reach the medulla and may have only capillaries possibly originating from the cortex.

By embryonic day 20, a three-dimensional KPN develops from the elevated keratin-staining spots and the "dilation" of the PS forms the KNA. Thus, after hatching, the thymic medulla, recognized by HE staining, consists of the KPN connected to the cortical epithelial cells and of KNA multiply bound to the PSs (Fig. 1c).
Fig. 1 a-d Cytokeratin immunostaining. a Developing thymus of a 16-day-old chicken embryo. The areas of elevated cytokeratin staining (arrows) are in close association with the primary septae $(P S)$. b Developing thymus of a 20-dayold chicken embryo. The epithelial branches and their club-shaped enlargements are separated by PS, which cannot be distinguished from the keratinnegative area (KNA; stars). The increased keratin staining (arrows) indicates the rudiment of the keratin-positive network (KPN; $C$ cortex). c Developing thymus of a 6-week-old chicken. The PS is dilated (dotted outline) and continuous with the KNA of the thymic medulla. PS serves as a central stalk from which the four thymic medullary regions arise (SS secondary septae). d Thymus of an 8-week-old rabbit. KNA (star) and endogenousperoxidase-positive cells (EPCs; heavily stained deposits) are also present in mammals. e Thymus of a 6-week-old chicken, silver impregnation. The PSs at the cortico-medullary border spread and form reticular fibers in the KNA but not in the cortex $(C)$. Bar $200 \mu \mathrm{m}$

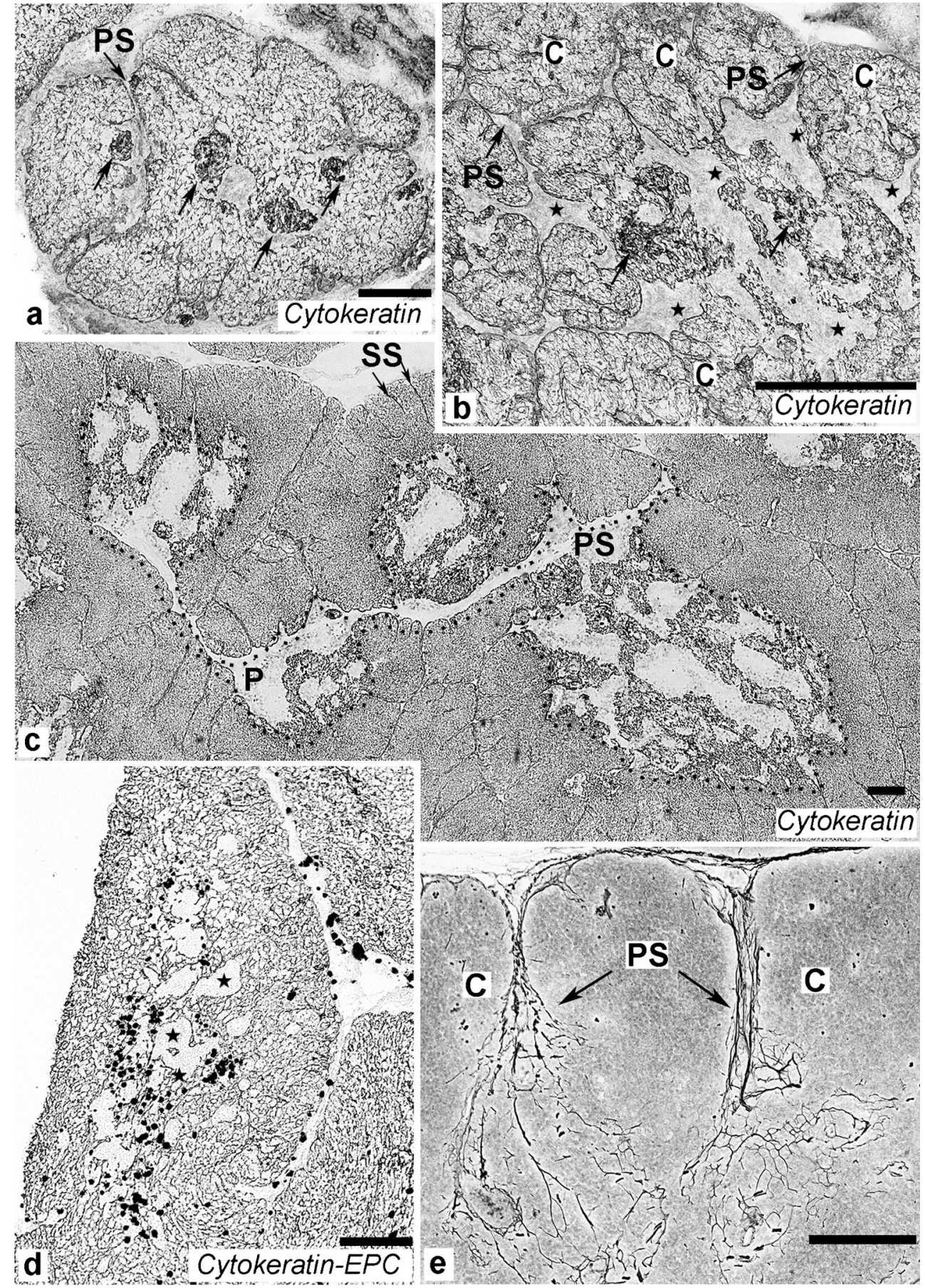


Morphometric analysis of thymuses of 3- and a 6-weekold chickens revealed that the volumetric ratio of the cortex and medulla was approximately 3 to 2 . In the 3 -week-old chicken, the volume of the KPN and KNA was $57 \%$ and $43 \%$, respectively, whereas these values in the 6-week-old chicken proved to be $53 \%$ and $47 \%$ (Fig. 2). In mammals, namely in rabbit and rat (data not shown), the KPN and the KNA also exist but the elevated keratin expression in the medulla cannot be seen (Fig. 1d).

Silver impregnation revealed reticular fibers in the PSs and KNA (Fig. 1e) but not in the cortex and KPN. The presence of type III collagen, which is the basic fibrillar motif of the reticular fiber, in the PS and KNA confirmed the result of silver impregnation (Fig. 3a). The existence of reticular fibers indicates that, in the PSs and KNA, reticular connective tissue is formed that produces other ECM molecules, like fibronectin (Fig. 3b) and tenascin (Fig. 3c). Reticular cells are modified fibroblasts, which are highly versatile cells, capable of ECM production, the isolation of reticular fibers from T-cells, Bcells and DCs, the phagocytosis of foreign substances and occasionally antigen presentation. Vimentin intermediate filament is produced by the fibroblasts and reticular cells. Antivimentin immunostaining shows the classic histological pattern of thymic cortex and medulla, without distinguishing the KPN and KNA (Fig. 3d). After hatching, an increasing number of B-cells enters the thymic medulla, which shows a similar staining pattern as the HE and anti-vimentin. The density of B-lymphocytes and vimentin-positive cells varies; in some areas, the cell density is high, whereas other areas are almost devoid of cells (Fig. 3d, e).

The surface epithelium of the thymic lobe facing towards the capsule and the septae is covered by basal lamina indicated by the presence of laminin (Fig. 3f). At the appearance of the KPN, the basal lamina becomes discontinuous (Fig. 3f, g). Thus, the KNA carrying the arteries and veins is separated by a discontinuous basal lamina from the KPN (Fig. 3f, g). The marginal epithelial cells of the KPN, in addition to keratin, express vimentin intermediate filament (see below). Cells of Hassall's bodies (not shown) do not express vimentin (Minkó and Oláh 1996), like the inner cells of the KPN.
Administration of high-dose DM induces acute involution, which results in T-cell depletion and subsequently abrogation of the keratin pattern of the cortex (Fig. 3h). The entire lobe shows a medullary appearance and also elevated production of fibronectin (Fig. 3i) and tenascin (Fig. 3j).

A unique cell type of the KNA is the myoid cell, which occurs with a round and/or elongated shape (Fig. 4a) and expresses desmin intermediate filament (Fig. 4b). Antidesmin monoclonal antibody ( $\mathrm{mAb}$ ) is a convenient tool for the identification of myoid cells, which are single cells, like the smooth muscle cells. Myosin and actin filaments are occasionally organized in sarcomeres as in skeletal muscle, showing alternating light I and dark A bands (Fig. 4c).

The epithelial cyst is also a common structure of the KPN. The cyst is generally lined by microvilli that are highly regular, as in the small intestine and the cells secrete a mucus substance (Figs. 4d). The presence of microvilli, a terminal web, a cell junction at the lateral cell membrane (Fig. 4e) and the mucus substance inside the cyst indicate that the cystforming epithelial cells maintain their polarity, unlike the reticular epithelial cells.

We used chicken follicular dendritic cell (FDC)-specific $\mathrm{mAb} 74.3$ and transmission electron microscopy for the in vivo search for thymic DCs. In the KNA of the thymic medulla, mAb 74.3 immunocytochemistry revealed grouped or confluent positive reaction and scattered individual cells (Fig. 5a). Endogenous-peroxidase-positive cells (EPC) also form groups in the medulla (Olah et al. 1991). However, EPC histochemistry and $74.3 \mathrm{mAb}$ immunocytochemistry clearly indicated that the EPCs and 74.3-positive cells represented two different cell populations (Fig. 5a, inset). The EPCs occurred in both the KPN and KNA, unlike the 74.3positive DCs, which were localized in the center of the KNA (Fig. 5b) in close association with the blood vessels (Fig. 5c) indicated by smooth muscle actin (SMA) immunostaining. These 74.3-positive DCs expressed MHC II antigen (Fig. 5d, inset). The distribution of chB6-positive B-lymphocytes and 74.3-positive DCs showed a topographical pattern, namely, DCs occupied the center of the KNA (Fig. 5b) and Bcells formed a dense layer close to the surface of the KPN
Fig. 2 Volumetric illustration of thymic compartments
Volumetric illustration of thymic compartments
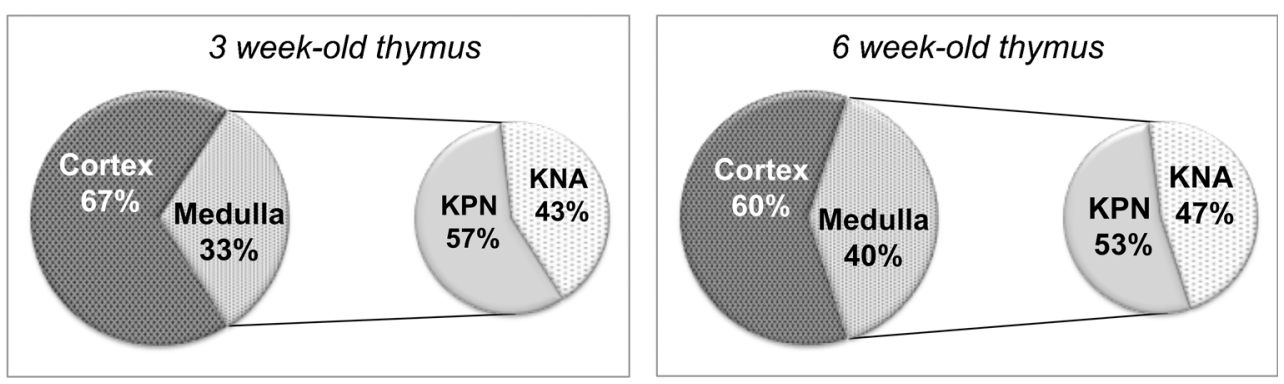

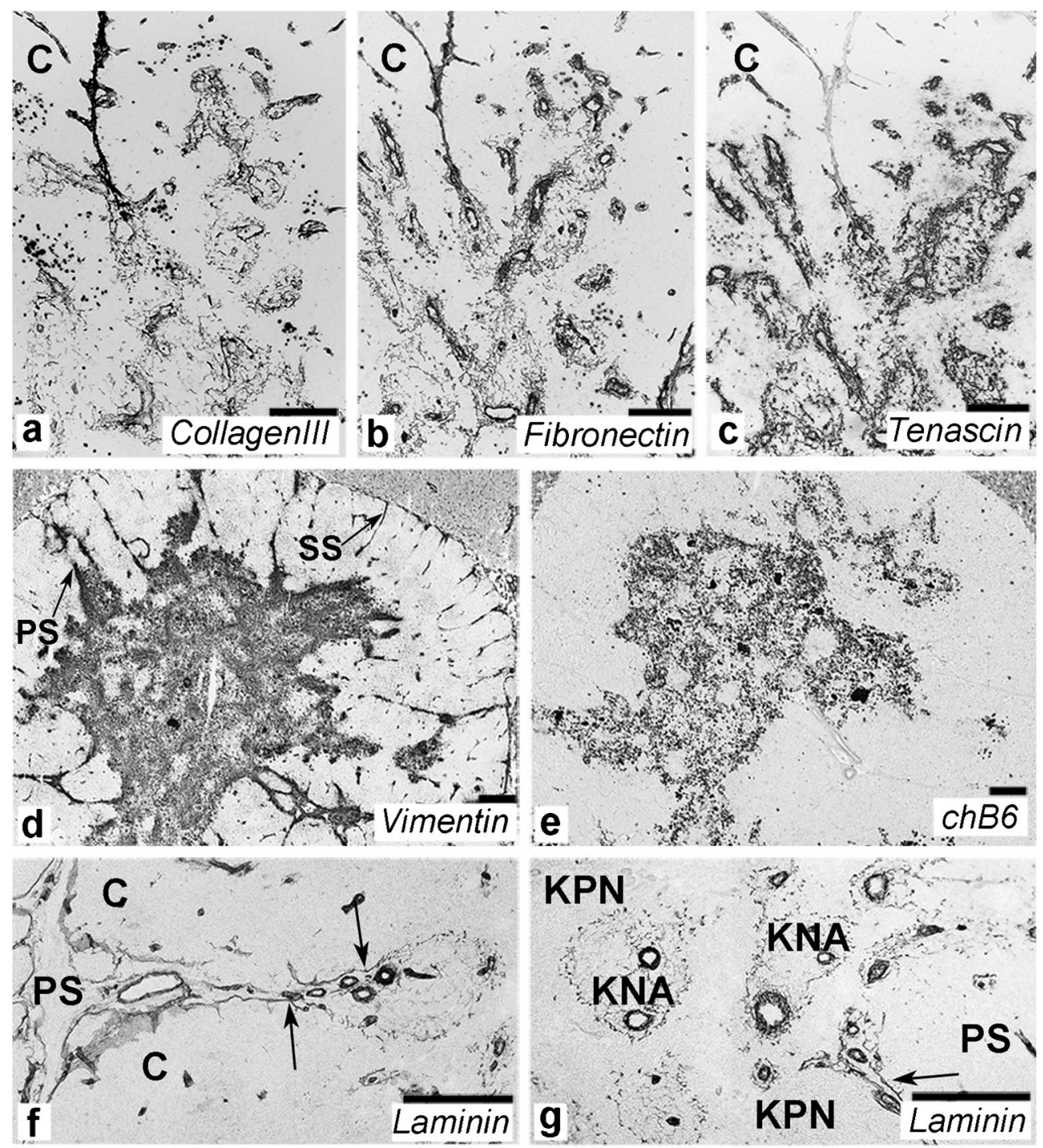
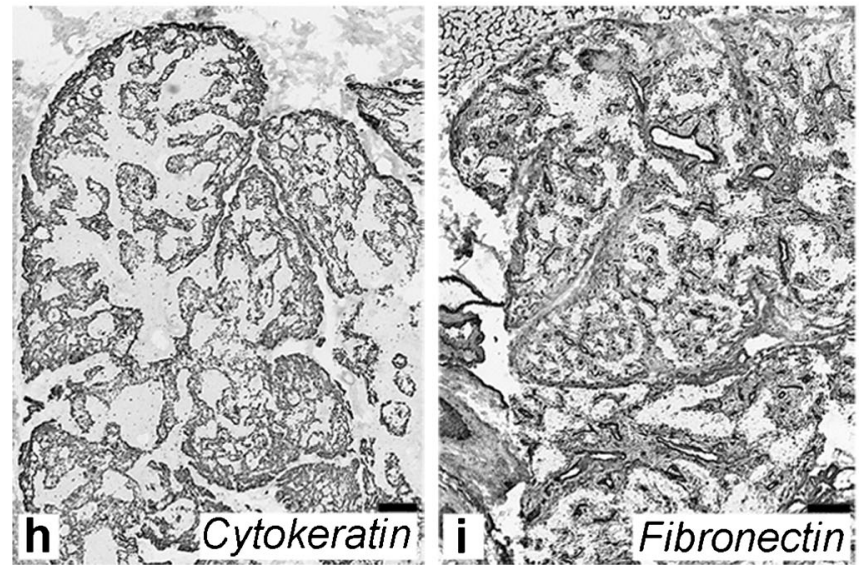

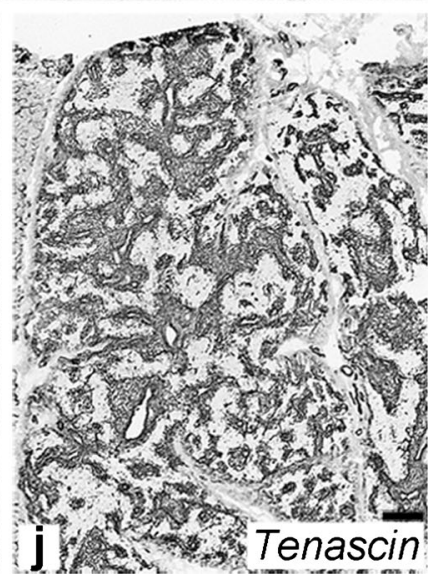

Fig. 3 Chicken thymus at 6 weeks of age. a Collagen III is the basic fibrillar molecule of reticular fibers. b A fine fibronectin network is restricted to the KNA of the thymic medulla. $\mathbf{c}$ Tenascin is also located in the KNA. d Anti-vimentin monoclonal antibody recognizes the entire medulla but the density of vimentin-positive cells varies. Vimentinpositive cells outline both the primary septum $(P S)$ and secondary septum $(S S)$. e Anti-chB6 immunostaining. B-cells are located only in the medulla. The distribution of B-cell density is inhomogeneous. f, $\mathbf{g}$ Antilaminin immunostaining. PS receives the thymic arteries and veins. The surface of the cortex $(C)$ is covered by a basal lamina that becomes discontinuous at the cortico-medullary border (arrows). $\mathrm{g}$ KNA with the blood vessels are outlined by the discontinuous basal lamina of the KPN (PS primary septum). $\mathbf{h}-\mathbf{j}$ Dexamethasone-treated chickens. h Anticytokeratin immunostaining. i Anti-fibronectin immunostaining. j Antitenascin immunostaining. The cortical staining pattern completely disappears and the entire thymic lobe resembles the medulla. Both extracellular matrix (ECM) molecules show an increase in the KNA. Bar $200 \mu \mathrm{m}$ 
Fig. 4 a Transmission electron micrograph of a myoid cell embracing a bunch of collagen fibers $(C O)$ in the KNA. b Myoid cells (arrows) are heavily stained by anti-desmin. c Semithin section stained by toluidin blue. Myosin and actin filaments are organized as in skeletal muscles. Migration of lymphocytes through the postcapillary (arrow). d Epithelial cells of a cyst are highly polarized. Apical surfaces of the cyst-forming epithelial cells are covered by microvilli and the lumen contains a medium-dense substance. e Higher magnification of the boxed area shown in $\mathbf{d}$. The apical part of the lateral cell membrane displays a cell junction (arrows). Bar $200 \mu \mathrm{m}(\mathbf{b}, \mathbf{c})$, $1 \mu \mathrm{m}(\mathbf{a}, \mathbf{d}, \mathbf{e})$
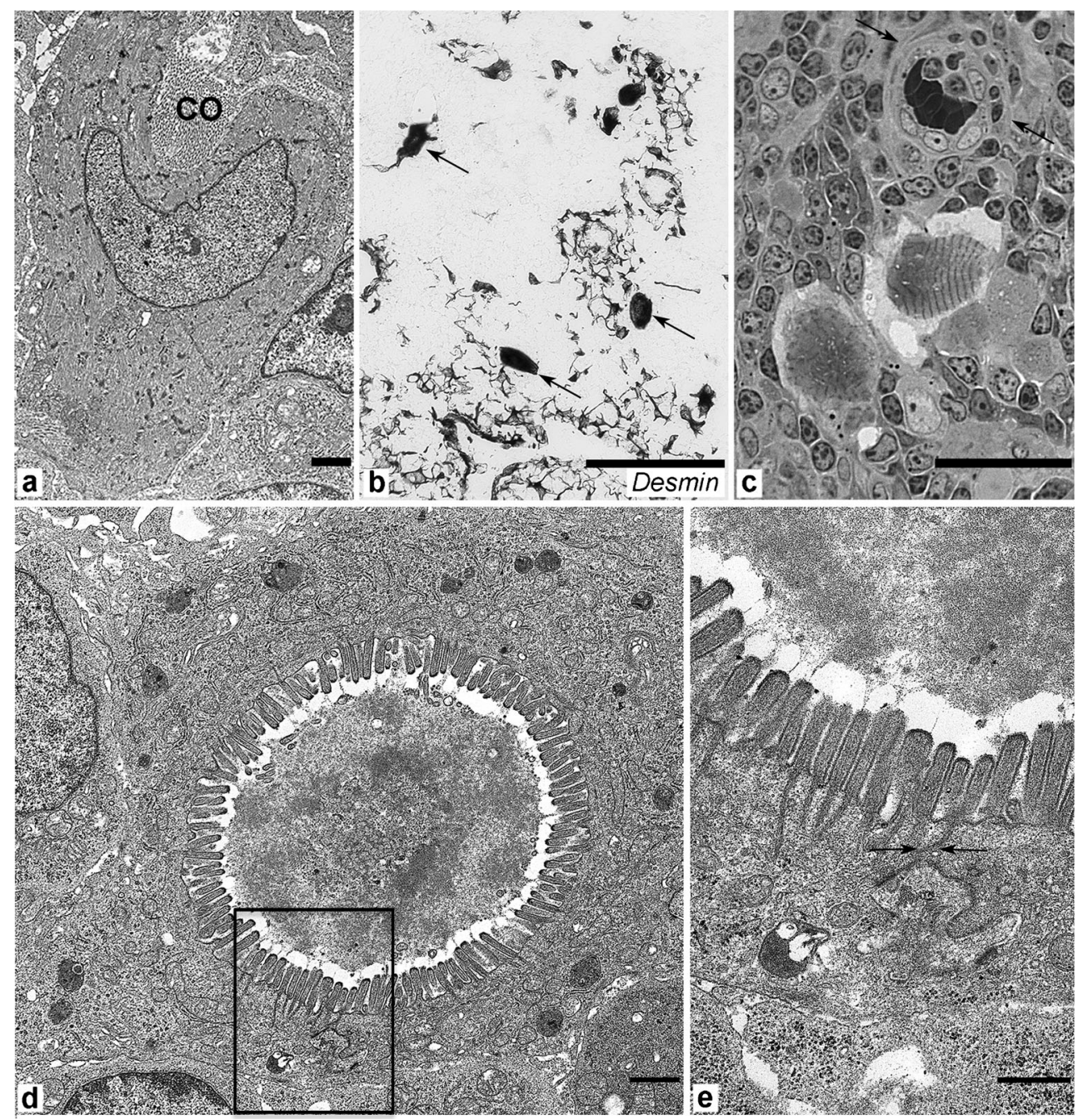

(Fig. 5e). The CD4 single-positive cells of the medulla were arranged mainly in the KNA (Fig. 6a, b), whereas the distribution of CD8 seemed to be the opposite (Fig. 6b, c).

Transmission electron microscopy of a thymic DC shows an excentrically located, irregularly shaped nucleus with nuclear-membrane-associated heterochromatin. The bulky cytoplasm produces a few short thick processes and highly electron dense, irregularly shaped and sized granules (Fig. 6d) that occasionally show a lamellated appearance (Fig. 6e).

\section{Discussion}

The thymus is the only lymphoid organ that is lobulated and develops like a gland (Fig. 7a). In a cross-section, the epithelial cord and the club-shaped enlargements appear as islets embedded in mesenchyme (Rodewald 2008; Rodewald et al. 2001) of neural crest origin. These experimental observations confirm the early histological findings, which described the thymic anlage as an epithelial island surrounded by mesenchyme (Maximov and Bloom 1938). The mesenchyme differentiates to PS separating the branches of the epithelial cord (Fig. 7a, b). One branch of the epithelial cord possibly develops from one epithelial progenitor cell. The club-shaped enlargements are the anlage of the future cortex, whereas the developing KNA seems to be dilations of the PSs. In this context, the PS can be regarded as a central stalk, from which the KNA of the medulla arises. Thus, the thymic medulla is divided for KPN and KNA. The close topographical connection between the PS and KNA suggests their common origin from cranial neural crest cells. In the KNA, the presence of myoid cells of skeletal muscle structure further supports the neural crest origin of KNA. Striated muscle normally can develop only from the myotomes of somites, the prechordal plate and cranial neural crest cells but not from the ectoderm and endoderm. The myogenic potency of the neural crest cells has been confirmed by several authors (Johnston et al. 1979; Nakano and Nakamura 1985; Yamashita and Sohal 1987). On the basis of experimental observations, the origin of the myoid 

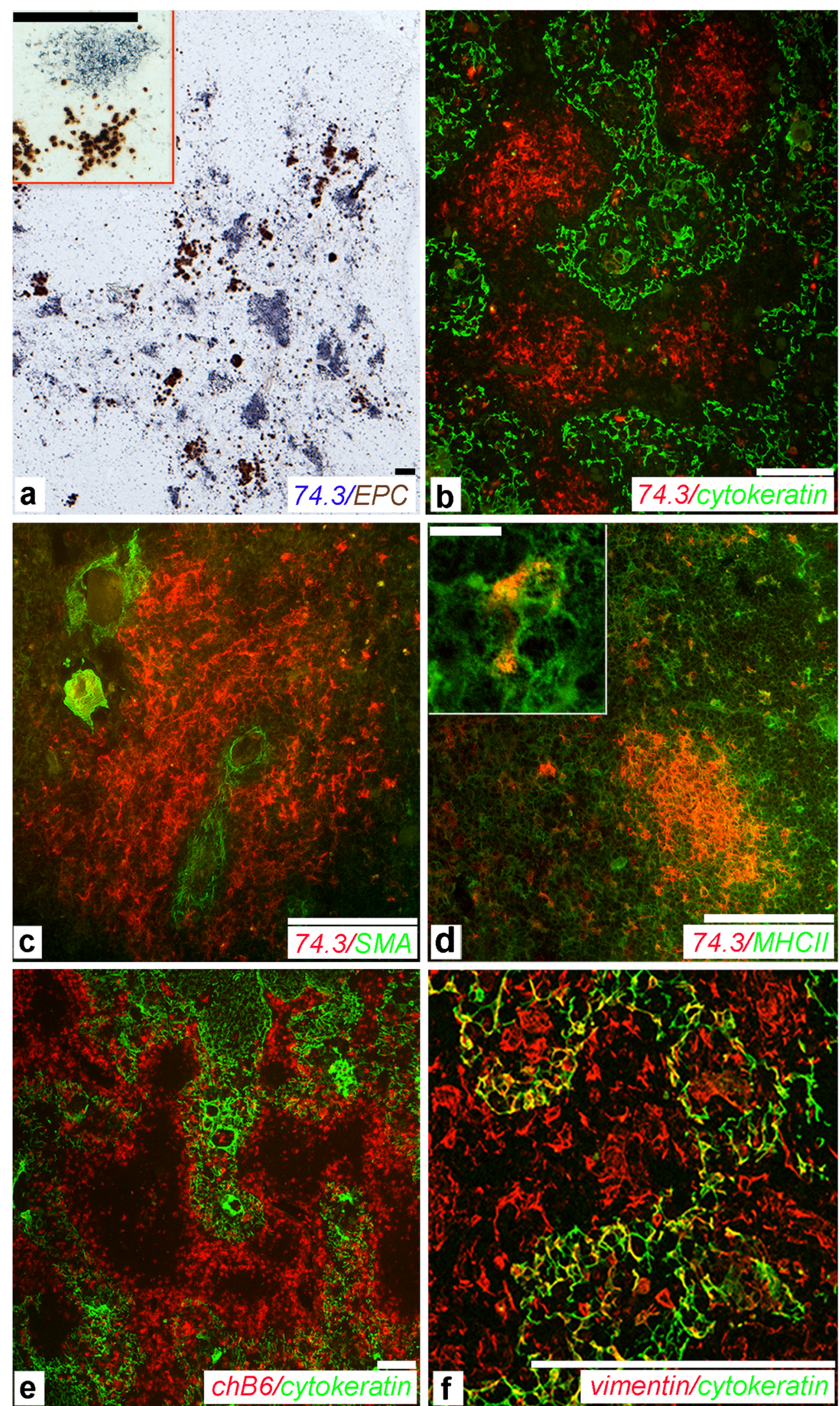

Fig. 5 a Both EPCs (brown) and 74.3-positive cells (blue) are grouped but are not co-localized (inset). b The 74.3-positive dendritic cells (DCs; red)

MHC II antigen (inset confocal micrograph) e The chB6 positive B-cells (red) accumulate in the KNA close to the surface of the KPN (green). $\mathbf{f}$ Confocal micrograph. Keratin (green) is co-localized with vimentin (red) in the marginal epithelial cells of the KPN (yellow). Bar $100 \mu \mathrm{m}$ 
Fig. 6 a-c Serial sections of a thymus with identical areas outlined. a CD4-positive cells accumulate in the KNA. b Anticytokeratin indicates the KNA. c CD8-positive cells occur in low numbers in the KNA. d

Transmission electron micrograph of a thymic DC. The irregularly shaped nucleus, a few cytoplasmic granules and stocky processes of ectoplasm are characteristic features of thymic DC (outlined). e The granules of DC occasionally show lamellated structures. Bar $200 \mu \mathrm{m}(\mathbf{a}-\mathbf{c})$, $1 \mu \mathrm{m}(\mathbf{d}, \mathbf{e})$
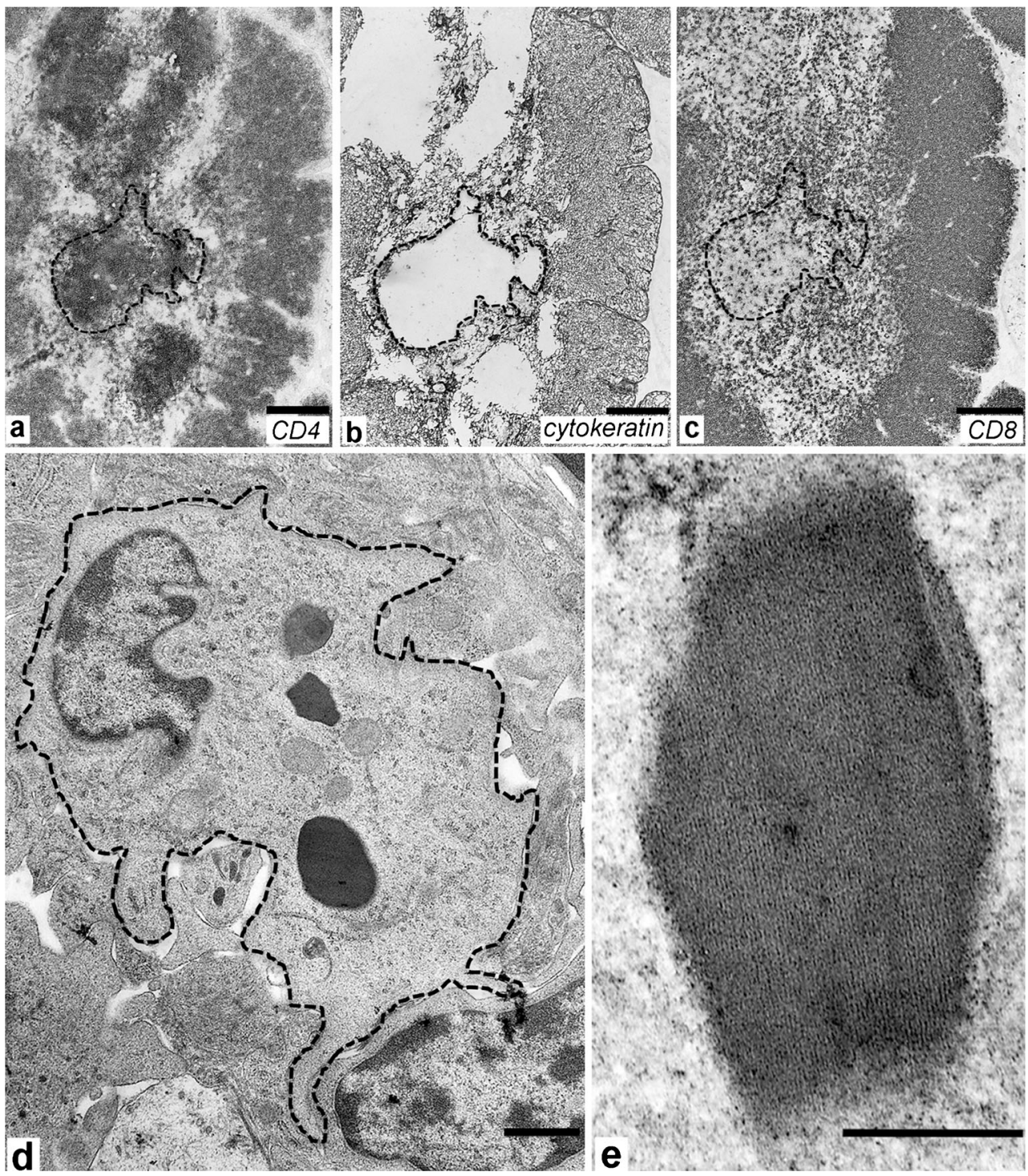

cells from neural crest cells has also been proposed (Nakamura and Ayer-Le Lièvre 1986) and might also indirectly confirm the neural crest origin of the KNA. However, Seifert and Christ (1990) reported that thymic myoid cells are derived from the axially located prechordal plate and not from mesencephalic or the rhombencephalic neural crest cells. These authors proposed that prechordal cells can contribute to the branchial arches from which the myoid cells can migrate into the thymic anlage. The presence of myoid cells, in addition to the pericytes in the KNA, indicates that the volume of KNA is much larger than the perivascular space. Morphometric analysis of keratin-stained serial sections confirmed that the KNA makes up about half the volume of the medulla, at least in the chicken (Fig. 2). Seifert and Christ (1990) suggested that the origin of the myoid cells is the prechordal plate. This theoreticaly means that the thymus develops not from three but from four different sources: (1) the branchial endodermal pouch, (2) the neural crest, (3) hematopoietic progenitors and (4) the prechordal plate. This might explain the highly heterogenic cellular composition of the thymic medulla allowing the high number of tissuespecific antigens for the induction of central tolerance (Dooley et al. 2005).

The KNA contains reticular fibers, including type III collagen, plus fibronectin and tenascin. Both fibronectin and tenascin are capable of influencing cell migration (Savino et al. 2000; 2004). Various concentrations of tenascin can regulate neural crest cell migration (Akbareian et al. 2013) and subsequently influence the effeciency of the autoreactive T-cell selection. The presence of reticular fibers at the corticomedullary border and in the inner medulla around the Hassall's bodies of human thymus has been shown by $\mathrm{Yu}$ and Lee (1993). The amount of reticular fibers and ECM components increases with age during physiological (Savino et al. 1993) and acute (Lannes-Vieira et al. 1991; Gameiro et al. 2010) involutions. Our experimental results, in which 
Fig. 7 a, b Representation of the development of the thymic anlage. a Branches of the epithelial cord grow into the mesenchyme and form the PS. On the surface of the cortex and PS, the basal lamina is continuous (black line). b Relationship between the medulla and PS. The surface of the KPN is covered by a discontinuous basal lamina (dashed line). c Cellular organization of KNA. B-cells accumulate along the borderline of the KPN and KNA, whereas in the center of the KNA, the DCs are localized around the blood vessels
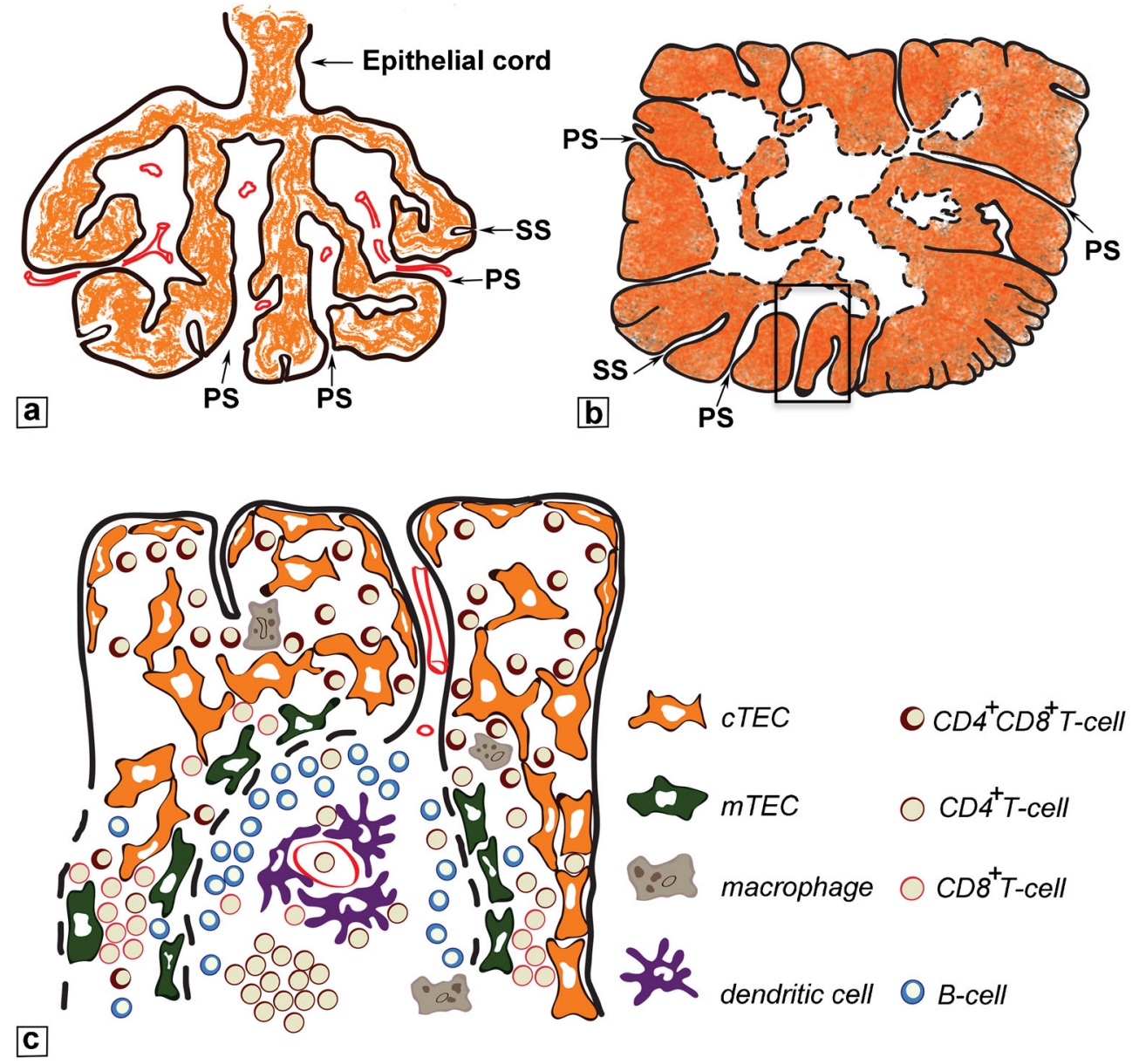

acute involution was induced by DM, confirmed the increase of the ECM of the KNA. In contrast to the cortex and the KPN, which have an epithelial reticulum of endodermal origin, the KNA is composed of reticular connective tissue, which is less sensitive to cortisone treatment than the central lymphoid organs. DM treatment results in severe T-cell depletion but the basic structural elements, namely, the endodermal epithelial cells, the thymic capsule and PSs of neural crest origin survive. After DM treatment, the entire thymic lobe becomes "medulla", i.e., consists in the KPN and KNA. These thymic structural changes further support the neural crest origin of KNA.

In birds, the thymus and the bursa of Fabricius are the primary lymphoid organs contributing to the development of T- and B-lymphocytes, respectively. Not only the histological structure (reticular connective tissue) of the KNA is comparable to that of the cortex of the bursal follicle but functionally, both structures serve for cell migration between primary and secondary lymphoid organs. Therefore, both the KNA of the thymic medulla and the cortex of the bursal follicles can be regarded as a transit zone of primary lymphoid organs. Lymphocytes leaving these transit zones reach the gutassociated lymphoid tissues (the main peripherial lymphoid organs of chickens) via high endothelial venules (HEV), which are regulated by CD11 $\mathrm{c}^{+}$DCs (Moussion and Girard 2011). The thymic postcapillaries are not HEV but an extensive cell migration takes place between the blood circulation and the KNA. Large numbers of immunologically matured Tcells enter the circulation and after hatching, a significant number of B-cells migrates into the thymic medulla. Double-immunolabeling of keratin and B-cells shows that the majority of the chB6-positive B-lymphocytes line up along the KPN, whereas the postcapillaries together with the 74.3 and MHC II double-positive DCs are localized in the center of the KNA (Fig. 7c). The topographical arrangement of B-cells and DCs indicates some cell-sorting mechanism in the KNA.

The blood-thymus barrier exists only in the cortex (Raviola and Karnovsky 1972) and therefore, thymocyte maturation takes place in an antigen-free microenviroment. The cortical barrier is formed by (1) endothelial cells and their basal lamina of the cortical capillaries and (2) the cortical surface epithelial cells and their basal lamina. However, there is neither a histological nor an anatomical explanation for the differences in the barrier between the cortex and medulla. The cortical epithelial cells produce only keratin filaments, unlike the KPN 
surface epithelial cells, which express both keratin and vimentin intermediate filaments. The epithelial cells of the KPN have a discontinuous basal lamina, which breaks up the blood-thymus barrier. Thus, the anti-laminin staining pattern and the colocalization of keratin and vimentin in the surface cells of the KPN seem to be the first cytological sign and molecular marker for the difference of the blood-thymus barrier between the cortex and medulla and support epithelial plasticity (De Souza and Savino 1993).

After DM treatment, the cortex drastically shrinks and the entire lobe resembles the medulla. Subsequently, the classical cortico-medullary border alters, actually disappearing and the marginal epithelial cells of the KPN only occasionaly express vimentin, unlike the untreated normal state. Therefore, the differentiation and/or plasticity of the marginal epithelial cells of the KPN are lost and the KNA is enlarged. Similar morphological events occur in mammals during age-related thymic involution, namely the expansion of the perivascular space (Gameiro et al. 2010). Infection by human immunodeficiency virus (HIV) is followed by thymic involution and histological disintegration of the cortico-medullary border (Napolitano et al. 2002). Similar experimental observations have been made after parasitic infection (Brito et al. 2003; Gameiro et al. 2010; Pérez et al. 2012) and malnutrition (Savino et al. 2007). These histological alterations are subsequently associated with increased subsceptibility to autoimmune disease and cancer development (Lynch et al. 2009). Notably, once DM treatment ceases, the thymus soon regenerates, indicating that either cortical progenitor cells survive the treatment or the cortical and medullary epithelial cells have a common progenitor, as proposed earlier (Bennett et al. 2002; Farr et al. 2002; Bleul et al. 2006; Rossi et al. 2007).

The Hassall's corpuscles (not shown) and cysts (Vroegindeweij et al. 2010) are other characteristic structures of the thymic medullary epithelium. Hassall's bodies are formed in the inner KPN and their cells do not express vimentin intermediate filament (Minkó and Oláh 1996), like the inner cells of the KPN. Possibly, the colocalization of keratin and vimentin in the marginal epithelial cells of the KPN represents epithelial plasticity (De Souza and Savino 1993) and the final differantiation stage, which is necessary for the function of the thymic medullary epithelial cells. The cells of the Hassall's body and the inner epithelial cells of the KPN possibly do not "reach" the final differentiation stage and therefore, these internal epithelial cells are aggregated (forming Hassall's bodies), keratinized and die. In this context, the Hassall's bodies and the vimentin-negative inner cells of the KPN have an identical structure and are functionally inert elements of the thymus. The "senescent" Hassall's bodies are invaded by vimentinpositive scavenger cells, such as heterophyl granulocytes and macrophages, which phagocytose, digest and eliminate the Hassall's bodies. The cysts develop from the epithelial progenitors but their development is stopped at an early stage (Dooley et al. 2005) and therefore, the epithelial cells of the cysts maintain their polarized shape; this results in the absence of cross-talk between epithelial cells and T-cells (Klug et al. 2002; Rossi et al. 2007) and mesenchymal cells (Jenkinson et al. 2007; Itoi et al. 2007; Neves et al. 2012). We propose that the formation of both cysts and Hassall's bodies represents "developmental malformation" of the medullary epithelial cells, namely, the differentiation of epithelial progenitors is arrested in early and late stages, repectively.

The search for thymic DCs with a dendritic-specific monoclonal antibody (mAb 74.3) by immuno-electron microscopy was less successful but our previous studies have demonstrated irregular, highly electron dense granules with a lamellated inner structure in the FDCs of the germinal center of the caecal tonsil (Olah and Glick 1979). In the center of the KNA, mAb 74.3 immuncytochemistry visualized thymic DCs that express MHC II antigen. Thus, on the basis of the topographical location of mAb-74.3-positive cells, their MHC class II antigen expression and the presence of lamellated cytoplasmic granules, we indentified the avian thymic DC in vivo. On the basis of these microscopical landmarks, conventional transmission electron microscopy revealed DC-like cells in the medulla. This is the first time that avian thymic DCs have been observed at the electron microscopic level.

In conclusion, anti-cytokeratin immunostaining shows that the thymic medulla consists in two regions. (1) The KPN possesses marginal epithelial cells that, in addition to keratin, express vimentin intermediate filaments and produce a discontinuous basal lamina. These cytological features might explain the absence of the blood-thymus barrier in the medulla. (2) The KNA is a dilation of the PS of cranial neural crest origin. The functional cortico-medullary border induces central tolerance and eliminates autoreactive T-cells in the border between the KPN and KNA. This study also indicates that the borderline of the KPN and KNA extends the functional cortico-medullary border. Early and late arrest of the differentiation of thymic progenitor cells results in medullary cysts and Hassall's body formation, respectively.

Acknowledgments The authors thank Jutka Fügedi, Zsuzsa Vidra and Mária Bakó for laboratory assistance. Antibodies for tenascin, fibronectin, laminin, collagen type III, desmin, vimentin and MHC II were obtained from the Developmental Studies Hybridoma Bank, developed under the auspices of the NICHD and maintained by the University of Iowa, USA.

\section{References}

Akbareian SE, Nagy N, Steiger CE, Mably JD, Miller SA, Hotta R, Molnar D, Goldstein AM (2013) Enteric neural crest-derived cells promote their migration by modifying their microenvironment through tenascin-C production. Dev Biol 382:446-456 
Bennett AR, Farley A, Blair NF, Gordon J, Sharp L, Blackburn CC (2002) Identification and characterization of thymic epithelial progenitor cells. Immunity 16:803-814

Bleul CC, Corbeaux T, Reuter A, Fisch P, Mönting JS, Boehm T (2006) Formation of a functional thymus initiated by a postnatal epithelial progenitor cell. Nature 441:992-996

Bockman DE, Kirby ML (1984) Dependence of thymus development on derivatives of the neural crest. Science 223:498-500

Boyd RL, Wilson TJ, Bean AG, Ward HA, Gershwin ME (1992) Phenotypic characterization of chicken thymic stromal elements. Dev Immunol 2:51-66

Brito VN, Souto PC, Cruz-Höfling MA, Ricci LC, Verinaud L (2003) Thymus invasion and atrophy induced by Paracoccidioides brasiliensis in BALB/c mice. Med Mycol 41:83-87

De Souza LR, Savino W (1993) Modulation of cytokeratin expression in the hamster thymus: evidence for a plasticity of the thymic epithelium. Dev Immunol 3:137-146

Dooley J, Erickson M, Farr AG (2005) An organized medullary epithelial structure in the normal thymus expresses molecules of respiratory epithelium and resembles the epithelial thymic rudiment of nude mice. J Immunol 175:4331-4337

Farley AM, Morris LX, Vroegindeweij E, Depreter ML, Vaidya H, Stenhouse FH, Tomlinson SR, Anderson RA, Cupedo T, Cornelissen JJ, Blackburn CC (2013) Dynamics of thymus organogenesis and colonization in early human development. Development 140:2015-2026

Farr AG, Dooley JL, Erickson M (2002) Organization of thymic medullary epithelial heterogeneity: implications for mechanisms of epithelial differentiation. Immunol Rev 189:20-27

Foster K, Sheridan J, Veiga-Fernandes H, Roderick K, Pachnis V, Adams R, Blackburn C, Kioussis D, Coles M (2008) Contribution of neural crest-derived cells in the embryonic and adult thymus. J Immunol 180:3183-3189

Gameiro J, Nagib P, Verinaud L (2010) The thymus microenvironment in regulating thymocyte differentiation. Cell Adh Migr 4:382-390

Gill J, Malin M, Sutherland J, Gray D, Hollander G, Boyd R (2003) Thymic generation and regeneration. Immunol Rev 195:28-50

Gordon J, Manley NR (2011) Mechanisms of thymus organogenesis and morphogenesis. Development 138:3865-3878

Guillemot FP, Oliver PD, Peault BM, Le Douarin NM (1984) Cells expressing Ia antigens in the avian thymus. J Exp Med 160:18031819

Igyártó BZ, Nagy N, Magyar A, Oláh I (2008) Identification of the avian B-cell-specific Bu-1 alloantigen by a novel monoclonal antibody. Poult Sci 87:351-355

Itoi M, Tsukamoto N, Yoshida H, Amagai T (2007) Mesenchymal cells are required for functional development of thymic epithelial cells. Int Immunol 19:953-964

Jenkinson WE, Jenkinson EJ, Anderson G (2003) Differential requirement for mesenchyme in the proliferation and maturation of thymic epithelial progenitors. J Exp Med 198:325-332

Jenkinson WE, Rossi SW, Parnell SM, Jenkinson EJ, Anderson G (2007) PDGFRalpha-expressing mesenchyme regulates thymus growth and the availability of intrathymic niches. Blood 109:954-960

Johnston MC, Noden DM, Hazelton RD, Coulombre JL, Coulombre AJ (1979) Origins of avian ocular and periocular tissues. Exp Eye Res 29:27-43

Klug DB, Carter C, Crouch E, Roop D, Conti CJ, Richie ER (1998) Interdependence of cortical thymic epithelial cell differentiation and T-lineage commitment. Proc Natl Acad Sci U S A 95:11822-11827

Klug DB, Carter C, Gimenez-Conti IB, Richie ER (2002) Cutting edge: thymocyteindependent and thymocyte-dependent phases of epithelial patterning in the fetal thymus. J Immunol 169:2842-2845

Krutsay M (1980) Szövettani technika. Medicina, Budapest

Lannes-Vieira J, Dardenne M, Savino W (1991) Extracellular matrix components of the mouse thymus microenvironment: ontogenetic studies and modulation by glucocorticoid hormones. J Histochem Cytochem 39:1539-1546

Le Douarin N (1967) Early determination of the anlagen of the thyroid and thymus glands in the chick embryo. CR Acad Sci Hebd Seances Acad Sci D 264:940-942

Le Douarin NM, Dieterlen-Lièvre F, Oliver PD (1984) Ontogeny of primary lymphoid organs and lymphoid stem cells. Am J Anat 170:261-299

Le Lièvre CS, Le Douarin NM (1975) Mesenchymal derivatives of the neural crest: analysis of chimaeric quail and chick embryos. J Embryol Exp Morphol 34:125-154

Lynch HE, Goldberg GL, Chidgey A, Van den Brink MR, Boyd R, Sempowski GD (2009) Thymic involution and immune reconstitution. Trends Immunol 30:366-373

Maximov AA, Bloom W (1938) A textbook of histology, 3rd edn. Saunders, Philadelphia London

Minkó K, Oláh I (1996) Expression of intermediate filaments and Ncadherin adhesion molecule in the thymus of domesticated birds. Acta Biol Hung 47:323-340

Moussion C, Girard JP (2011) Dendritic cells control lymphocyte entry to lymph nodes through high endothelial venules. Nature 479:542-546

Müller SM, Stolt CC, Terszowski G, Blum C, Amagai T, Kessaris N, Iannarelli P, Richardson WD, Wegner M, Rodewald HR (2008) Neural crest origin of perivascular mesenchyme in the adult thymus. J Immunol 180:5344-5351

Nakamura H, Ayer-Le Lièvre C (1986) Neural crest and thymic myoid cells. Curr Top Dev Biol 20:111-115

Nakano KE, Nakamura H (1985) Origin of the irideal striated muscle in birds. J Embryol Exp Morphol 88:1-13

Napolitano LA, Lo JC, Gotway MB, Mulligan K, Barbour JD, Schmidt D, Grant RM, Halvorsen RA, Schambelan M, McCune JM (2002) Increased thymic mass and circulating naive CD4 T cells in HIV-1infected adults treated with growth hormone. AIDS 16:1103-1111

Neves H, Dupin E, Parreira L, Le Douarin NM (2012) Modulation of Bmp4 signalling in the epithelial-mesenchymal interactions that take place in early thymus and parathyroid development in avian embryos. Dev Biol 361:208-219

Noden DM (1978) The control of avian cephalic neural crest cytodifferentiation. II. Neural tissues. Dev Biol 67:313-329

Noden DM (1983) The embryonic origins of avian cephalic and cervical muscles and associated connective tissues. Am J Anat 168:257-276

Olah I, Glick B (1979) Structure of the germinal centers in the chicken caecal tonsil: light and electron microscopic and autoradiographic studies. Poult Sci 58:195-210

Olah I, Kendall C, Glick B (1991) Endogenous peroxidase- and vimentinpositive cells accumulate at the corticomedullary border of the chicken thymus. Poult Sci 70:1144-1152

Pérez AR, Berbert LR, Lepletier A, Revelli S, Bottasso O, Silva-Barbosa SD, Savino W (2012) TNF- $\alpha$ is involved in the abnormal thymocyte migration during experimental Trypanosoma cruzi infection and favors the export of immature cells. PLoS One 7:e34360

Raviola E, Karnovsky MJ (1972) Evidence for a blood-thymus barrier using electronopaque tracers. J Exp Med 136:466-498

Rodewald HR (2008) Thymus organogenesis. Annu Rev Immunol 26: 355-388

Rodewald HR, Paul S, Haller C, Bluethmann H, Blum C (2001) Thymus medulla consisting of epithelial islets each derived from a single progenitor. Nature 414:763-768

Rossi SW, Chidgey AP, Parnell SM, Jenkinson WE, Scott HS, Boyd RL, Jenkinson EJ, Anderson G (2007) Redefining epithelial progenitor potential in the developing thymus. Eur $\mathrm{J}$ Immunol 37:2411-2418

Savino W, Villa-Verde DM, Lannes-Vieira J (1993) Extracellular matrix proteins in intrathymic T-cell migration and differentiation? Immunol Today 14:158-161 
Savino W, Dalmau SR, Dealmeida VC (2000) Role of extracellular matrix-mediated interactions in thymocyte migration. Dev Immunol 7:279-291

Savino W, Mendes-Da-Cruz DA, Smaniotto S, Silva-Monteiro E, VillaVerde DM (2004) Molecular mechanisms governing thymocyte migration: combined role of chemokines and extracellular matrix. J Leukoc Biol 75:951-961

Savino W, Dardenne M, Velloso LA, Dayse Silva-Barbosa S (2007) The thymus is a common target in malnutrition and infection. Br J Nutr 98 (Suppl 1):S11-S16

Seifert R, Christ B (1990) On the differentiation and origin of myoid cells in the avian thymus. Anat Embryol (Berl) 181:287-298

Tschanz S, Schneider JP, Knudsen L (2014) Design-based stereology: planning, volumetry and sampling are crucial steps for a successful study. Ann Anat 196:3-11
Van Ewijk W, Rouse RV, Weissman IL (1980) Distribution of H-2 microenvironments in the mouse thymus. Immunoelectron microscopic identification of I-A and H-2K bearing cells. J Histochem Cytochem 28:1089-1099

Venzke WG (1952) Morphogenesis of the thymus of chicken embryos. Am J Vet Res 13:395-404

Vroegindeweij E, Crobach S, Itoi M, Satoh R, Zuklys S, Happe C, Germeraad WT, Cornelissen JJ, Cupedo T, Holländer GA, Kawamoto H, Ewijk W van (2010) Thymic cysts originate from Foxn1 positive thymic medullary epithelium. Mol Immunol 47: 1106-1113

Yamashita T, Sohal GS (1987) Embryonic origin of skeletal muscle cells in the iris of the duck and quail. Cell Tissue Res 249:31-37

Yu E, Lee I (1993) Reticular network of the human thymus. J Korean Med Sci 8:431-436 